\title{
Washout Control for Manual Operations
}

\author{
Takashi Takimoto, Student Member, IEEE, Shigeru Yamamoto, Member, IEEE, and Hiroshi Oku, Member, IEEE
}

\begin{abstract}
It is known that limitations of human accuracy in manual manipulation hinder the quality of work performed by human operators of manual control systems. Indeed, movements of operators are apt to cause undesirable vibrations in manual control systems. In this paper, we propose a new operator-support control scheme for suppressing harmful oscillatory motions in such systems without disturbing human operator's manipulation. The proposed scheme is based on the fact that steady-state blocking zeros of a feedback controller do not affect the steady-state control input. A finite-dimensional feedback controller with steady-state blocking zeros, called a washout controller in this paper, plays the central role in support for operator's manipulation. However, the dynamics of a manual control system may become different significantly from its initial model used for the design of an initial washout controller when it is applied to the manual control system. Such difference can result in poor performance of operator-support control. In order to improve it, an iterative procedure is presented for redesign of washout controllers based on closed-loop subspace identification. Closed-loop identification is performed to refine the model for the control design, and then a more sophisticated washout controller is obtained using the identified model. The effectiveness of the proposed scheme is demonstrated by an experiment on manual control of an inverted pendulum.
\end{abstract}

Index Terms-Blocking zeros, closed-loop identification, manmachine systems, vibration suppression, washout control.

\section{INTRODUCTION}

$\mathbf{T}$ HIS PAPER concerns operator-support control for a class of manual control systems. The operator-support control is useful to human operators for manipulation of unstable objects. Riding a bicycle and piloting an aircraft are typical examples of manual operations of unstable objects. In manual control systems, a crucial issue underlies undesirable vibration caused by quivering movements of human operators, due to limitations of human accuracy in manual manipulation [1]-[3]. Therefore, it is important to suppress undesirable vibration effectively during manual manipulation in order to improve control performance and to reduce operators' workload.

In operator-support control, it is important not to hinder manual manipulation intended by human operators. There are several methods of suppressing harmful vibration in manual control systems without disturbing manual manipulation, for

Manuscript received May 25, 2007; revised September 12, 2007. Manuscript received in final form January 3, 2008. First published August 22, 2008; current version published October 22, 2008. Recommended by Associate Editor K. J. Hunt.

T. Takimoto is with the Department of Systems Innovation, Graduate School of Engineering Science, Osaka University, Osaka 560-8531, Japan (e-mail: takimoto@moccos.ec.t.kanazawa-u.ac.jp).

S. Yamamoto is with Graduate School of Natural Science and Technology, Kanazawa University, Ishikawa 920-1192, Japan (e-mail: shigeru@t. kanazawa-u.ac.jp).

H. Oku is with the Department of Electronics, Information and Communication Engineering, Osaka Institute of Technology, Osaka 535-8585, Japan (e-mail: oku@elc.oit.ac.jp).

Digital Object Identifier 10.1109/TCST.2008.917871

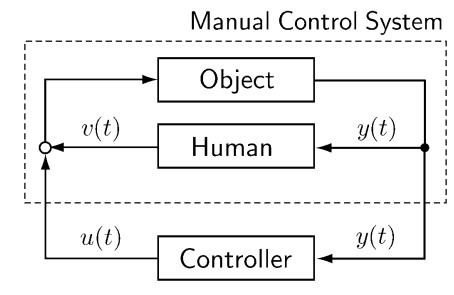

Fig. 1. Manual control system with the proposed operator-support controller.

example, a feedback configuration of delayed feedback control [4] and washout filters [5]-[7]. An attractive feature of these schemes is that the feedback controller has steady-state blocking zeros. Steady-state blocking zeros mean that the closed-loop system has a blocking zero at zero frequency. Therefore, a feedback controller with steady-state blocking zeros does not affect the steady-state control input. In other words, such a controller can eliminate a steady-state bias which can disturb operator's manipulation [8].

In [8], an operator-support control scheme has been developed based on the technique used in delayed feedback control. Although it has unveiled the effectiveness of the steady-state blocking zeros, the design procedure of the operator-support control scheme is burdensome due to infinite-dimensionality of the closed-loop system which is inherited from delayed feedback control (see [9] and [10] for the detailed design method for delayed feedback controllers).

In [5]-[7], the so-called washout filter has been introduced into finite-dimensional feedback controllers. The washout filter can be regarded as a high pass filter for eliminating the steadystate input. Washout filter aided controllers have been applied to many real-life systems, e.g., power systems [11].

In this paper, we will propose a finite-dimensional feedback controller with steady-state blocking zeros, called a washout controller. The proposed washout controller is an extension of washout filter aided controllers in the sense that less conservative conditions for controllers is given. The proposed operator-support control scheme is that a washout controller is added to a manual control system as an auxiliary feedback loop (see Fig. 1). However, it is difficult to obtain a good initial model of a manual control system used for the design of a washout controller due to uncertainty and inherent nonlinearity of human operation. Therefore, the initial model of a manual control system may contain large modeling errors. To overcome it, we will adopt the iterative procedure as follows: we will carry out closed-loop identification of the manual control system compensated by the initial washout controller, in order to obtain a better model of the manual control system used for the redesign of a washout controller. Particularly, we will use the SSARX method [12] for closed-loop identification. It is known as a closed-loop subspace identification method. 
To demonstrate the effectiveness of the proposed operatorsupport control scheme, an experiment on manual control of an inverted pendulum is performed. The system has a pendulum attached to a moving cart whose movement is controlled by a human operator's manipulation such that the pendulum is actively balanced and remains standing. The result of the experiment demonstrates that the proposed scheme successfully suppresses undesirable vibration in the manual control system.

This paper is organized as follows. First, in Section II, operator-support control for suppressing harmful vibration in manual control systems without disturbing manual manipulation is presented. Then, we propose a washout controller for the operator-support control scheme. In Section III, we give a parameterization of washout controllers in the case of continuous time and discrete time. In Section IV, we propose an iterative design method of washout controllers for operator-support control. In Section $\mathrm{V}$, we show experimental results which illustrate the effectiveness of the proposed scheme.

\section{A. Notation}

Throughout this paper, we use the following notation. When a matrix

$$
M=\left[\begin{array}{ll}
M_{11} & M_{12} \\
M_{21} & M_{22}
\end{array}\right]
$$

is divided into submatrices to form a square product with a matrix $N$ such as $M_{22} N$, the lower linear fractional transformation of $M$ and $N$ is defined by

$$
\begin{aligned}
& \qquad \mathcal{F}_{l}(M, N):=M_{11}+M_{12} N\left(I-M_{22} N\right)^{-1} M_{21} \\
& \text { if }\left|I-M_{22} N\right| \neq 0 \text {. }
\end{aligned}
$$

\section{PRoblem Statement}

\section{A. Manual Control Systems}

Suppose the manual control system depicted in the dashed rectangle in Fig. 1 can be described by the nonlinear differential equation

$$
\dot{x}(t)=f(x(t), u(t)) \quad y(t)=g(x(t), u(t))
$$

where $x(t) \in \Re^{n}$ is the state vector, $u(t) \in \Re^{r}$ is the auxiliary input vector for operator-support control, and $y(t) \in \Re^{m}$ is the measured output. $f$ and $g$ are assumed to be unknown smooth functions. The task of a human operator is to maintain the output $y(t)$ within a neighborhood of his/her desirable operating point $\tilde{y}$ by means of the regulation of the amount of operation $v(t) \in$ $\Re^{r}$.

On the manual control system, we make several assumptions as follows. The operating point $\tilde{y}$ is assumed to be a constant which is determined by the human operator. Assume that any numerical information of $\tilde{y}$ is not available a priori for operator-support control since no one but the human operator can understand it. Moreover, it is assumed that there exists an equilibrium point of the manual control system (1) for $u(t) \equiv 0, v(t) \equiv$ $\tilde{v}$, and $y(t) \equiv \tilde{y}$. That is, there exists a unique $\tilde{x}$ such that

$$
0=f(\tilde{x}, 0) \quad \tilde{y}=g(\tilde{x}, 0) \text {. }
$$

\section{B. Operator-Support-Control}

A crucial problem with the manual control system is that the measured output $y(t)$ may vibrate due to limitations of human accuracy and the nonlinear nature of the human operator. Our goal is to suppress the vibration. To this end, we will introduce an operator-support control scheme for manual control by means of feedback connection of an automatic controller to the manual control system (see Fig. 1). Let the automatic controller be represented as the following state space model:

$$
\dot{w}(t)=\hat{A} w(t)+\hat{B} y(t) \quad u(t)=\hat{C} w(t)+\hat{D} y(t)
$$

where $w(t) \in \Re^{\hat{n}}$ is the state vector of the controller and the control input $u(t)$ is applied to the manual operation $v(t)$.

The automatic controller is designed to locally stabilize the unknown equilibrium point $\tilde{x}$ in the manual control system (1) without changing its equilibrium point $\tilde{x}$. In other words, the stabilization should be achieved by unbiased input, i.e., $u(t)=0$ when $y(t)-\tilde{y}=0$. When the closed-loop system is stabilized, if the control input converges to a nonzero $\tilde{u}$, the human operator is forced to change his/her desired operation $\tilde{v}$ to $\tilde{v}-\tilde{u}$. In this way, the human operator is required to compensate the manual operation in order to eliminate the biased input $\tilde{u}$. This additional requirement imposes a burden of the human operator. To avoid it, operator-support control should be designed such that $\lim _{t \rightarrow \infty} u(t)=0$ and $\lim _{t \rightarrow \infty} y(t)=\tilde{y}$. From the assumption, however, the reference $\tilde{y}$ is not available for any automatic controller. Hence, the controller is required to stabilize the uncertain operating point. This may be satisfied if the coefficients of the controller satisfy the condition that there exists a $\tilde{w}$ such that

$$
\left[\begin{array}{l}
0 \\
0
\end{array}\right]=\left[\begin{array}{ll}
\hat{A} & \hat{B} \\
\hat{C} & \hat{D}
\end{array}\right]\left[\begin{array}{l}
\tilde{w} \\
\tilde{y}
\end{array}\right]
$$

for any constant $\tilde{y}$.

\section{Stabilization of Unknown Operating Point}

A salient feature of our control problem is stabilization of the unknown operating point. The feedback controller (2) is designed so that the unknown $\tilde{y}$ is locally asymptotically stabilized. The design is carried out based on the linearized model of the manual control system. In the vicinity of the unknown $\tilde{y}$, the manual control system is linearized as

$$
\begin{aligned}
\delta \dot{x}(t) & =A \delta x(t)+B u(t) \\
y(t) & =C \delta x(t)+D u(t)+\tilde{y}
\end{aligned}
$$

where $\delta x(t)=x(t)-\tilde{x}$ and

$$
\left[\begin{array}{ll}
A & B \\
C & D
\end{array}\right]=\left[\begin{array}{ll}
\frac{\partial f}{\partial x}(\tilde{x}, 0) & \frac{\partial f}{\partial u}(\tilde{x}, 0) \\
\frac{\partial g}{\partial x}(\tilde{x}, 0) & \frac{\partial g}{\partial u}(\tilde{x}, 0)
\end{array}\right] .
$$

(The parameters of the state-space model will be identified by methods in Section IV.) Define $P(s)=D+C(s I-A)^{-1} B$. Then, the closed-loop system of $P(s)$ and (2) is depicted in Fig. 2, where $K(s)=\hat{D}+\hat{C}(s I-\hat{A})^{-1} \hat{B}$. For the closed-loop system, the unknown operating point $\tilde{y}$ can be regarded as a step disturbance. Hence, the problem of stabilizing the unknown 


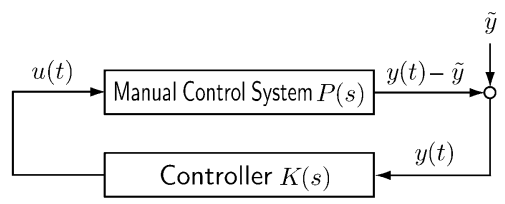

Fig. 2. Closed-loop system of the linearized system $P(s)$ around the unknown operating point $\tilde{y}$ in the manual control system and the proposed operator-support controller $K(s)$.

operating point $\tilde{y}$ can be cast into that of disturbance attenuation of the closed-loop system.

An important inherent property of controllers to stabilize unknown operating points is that their transfer functions have blocking zeros at zero frequency.

Definition 1: A transfer function matrix $K(s)$ (respectively, $K(z)$ for discrete-time systems) is said to have a steady-state blocking zero if $K(s)=0$ at $s=0$ (respectively, $K(z)=0$ at $z=1)$

If a feedback controller $K(s)$ with a steady-state blocking zero stabilizes the closed-loop system, the steady-state input always satisfies $\lim _{t \rightarrow \infty} u(t)=0$ because, from the final value theorem

$$
\begin{aligned}
\lim _{t \rightarrow \infty} u(t) & =\lim _{s \rightarrow 0} s U(s) \\
& =\lim _{s \rightarrow 0} s\{I-K(s) P(s)\}^{-1} K(s) \tilde{Y}(s) \\
& =\{I-K(0) P(0)\}^{-1} K(0) \tilde{y} .
\end{aligned}
$$

Therefore, the biased input can be removed by any feedback controller with $K(0)=0$ which stabilizes the manual control system.

Similarly, in discrete-time setting, blocking zeros play an important role in stabilization of an unknown fixed point of discrete-time systems. When we consider a linearized system around an unknown fixed point as

$$
\begin{aligned}
\delta x(k+1) & =A \delta x(k)+B u(k) \\
y(k) & =C \delta x(k)+D u(k)+\tilde{y}
\end{aligned}
$$

we stabilize it by a discrete-time controller $K(z)$ with its statespace realization

$$
\begin{aligned}
w(k+1) & =\hat{A} w(k)+\hat{B} y(k) \\
u(k) & =\hat{C} w(k)+\hat{D} y(k) .
\end{aligned}
$$

If $K(z)$ stabilizes (5) and has a steady-state blocking zero, then the steady-state input always satisfy $\lim _{k \rightarrow \infty} u(k)=0$. Because

$$
\begin{aligned}
\lim _{k \rightarrow \infty} u(k) & =\lim _{z \rightarrow 1}\left(1-z^{-1}\right) U(z) \\
& =\lim _{z \rightarrow 1}\left(1-z^{-1}\right)\{I-K(z) P(z)\}^{-1} K(z) \tilde{Y}(z) \\
& =\{I-K(1) P(1)\}^{-1} K(1) \tilde{y} .
\end{aligned}
$$

\section{Washout Control}

It is well known that delayed feedback control utilizes the difference between the current output and the delayed output to eliminate the biased input in the steady state. In fact, it was applied to the operator-support control problem in [8]. The transfer function of the delayed feedback controller can be factored $K(s)=K(s)^{\prime}\left(1-e^{-T s}\right)$, where $K(s)^{\prime}$ denotes a real rational transfer function. Hence, it has a steady-state blocking zero. In contrast to the simple control structure described here, the design procedure is complicated for practical use. In [5], to stabilize the continuous-time system with the unknown equilibrium point, a certainty equivalence adaptive control scheme was proposed. In [6] and [7], a design method of a washout filter aided feedback controller was discussed. The continuous-time washout filter aided feedback controller and the discrete-time one satisfy

$$
\begin{aligned}
\operatorname{det} \hat{A} & \neq 0, \hat{B}=-\hat{A}, \hat{D}=-\hat{C} \\
\operatorname{det}(I-\hat{A}) \neq 0, \hat{B} & =I-\hat{A}, \hat{D}=-\hat{C}
\end{aligned}
$$

respectively. The washout filter is a high-pass filter which is added to a feedback controller in order to eliminate the biased input in the steady state. However, (8) and (9) are sufficient conditions for the finite-dimensional controller with a steady-state blocking zero. These conditions are very conservative.

In this paper, we propose less conservative conditions for finite-dimensional controllers with a steady-state blocking zero. These controllers are shown in the following.

Definition 2: A continuous-time controller with a state-space realization (2) is called a washout controller if it satisfies the conditions

$$
\operatorname{det} \hat{A} \neq 0 \quad \hat{D}-\hat{C} \hat{A}^{-1} \hat{B}=0 .
$$

Definition 3: A discrete-time controller with a state-space realization (6) is called a discrete-time washout controller if it satisfies the conditions

$$
\operatorname{det}(I-\hat{A}) \neq 0, \hat{D}+\hat{C}(I-\hat{A})^{-1} \hat{B}=0 .
$$

In fact, the conditions (8) and (9), respectively, are sufficient for (10) and (11).

Lemma 1: If the feedback controller (2) is the washout controller, then it has a steady-state blocking zero.

Proof: It is obvious that the feedback controller (2) with the condition (10) satisfies $K(s)=\hat{D}+\hat{C}(s I-\hat{A})^{-1} \hat{B}=0$ at $s=0$.

Lemma 2: If the feedback controller (6) is a discrete-time washout controller, then it has the steady-state blocking zero.

Proof: Since the controller (6) with the condition (11) satisfies $K(z)=\hat{D}+\hat{C}(z I-\hat{A})^{-1} \hat{B}=0$ at $z=1$, we complete the proof.

\section{Full ORder PARAMETERIZATION OF WASHOUt CONTROLLERS}

In this section, we will give a full order parameterization of washout controllers. The term "full order" implies that the order of the controller is the same as that of the plant.

For the plants, we make an assumption of controllability and observability in this section. It can be relaxed to stabilizability and detectability. 


\section{A. Continuous-Time Washout Controller}

Let us suppose that a state-space realization of a continuoustime linear system be given as

$$
\dot{x}(t)=A x(t)+B u(t) \quad y(t)=C x(t)+D u(t) .
$$

Then, we have the following theorem for the full-order continuous-time washout controller.

Theorem 1: There exists a continuous-time washout controller stabilizing (12) if and only if $A$ is nonsingular. Moreover, when the above condition holds, a set of stabilizing washout controllers is given by

$$
\left[\begin{array}{ll}
\hat{A} & \hat{B} \\
\hat{C} & \hat{D}
\end{array}\right]=\mathcal{F}_{l}(J, Q)
$$

where $Q=-F A^{-1} L$

$$
\left.J=\left[\begin{array}{cc}
A+B F+L C+L D F & -L \\
F & 0
\end{array}\right]\left[\begin{array}{c}
B+L D \\
I \\
-D
\end{array}\right]\right]
$$

and $(F, L) \in \mathcal{S}:=\{(F, L) \mid A+B F$ and $A+L C$ are asymptotically stable, and $I+Q D$ is nonsingular. $\}$.

Proof: For notational brevity, we will introduce the notations as follows: If the closed-loop system with the controller (2) is well-posed (i.e., $I-D \hat{D}$ is invertible), it can be described as

$$
\left[\begin{array}{c}
\dot{x}(t) \\
\dot{w}(t)
\end{array}\right]=A_{c}\left[\begin{array}{c}
x(t) \\
w(t)
\end{array}\right]
$$

where

$$
\begin{aligned}
A_{c} & =\left[\begin{array}{ll}
A_{c 11} & A_{c 12} \\
A_{c 21} & A_{c 22}
\end{array}\right] \\
& :=\left[\begin{array}{cc}
A+B(I-\hat{D} D)^{-1} \hat{D} C & B(I-\hat{D} D)^{-1} \hat{C} \\
\hat{B}(I-D \hat{D})^{-1} C & \hat{A}+\hat{B}(I-D \hat{D})^{-1} D \hat{C}
\end{array}\right] .
\end{aligned}
$$

(Necessity) If the controller (2) stabilizes the closed-loop system and it is a washout controller, then $\operatorname{det}(I-D \hat{D}) \neq$ 0 , $\operatorname{det} \hat{A} \neq 0$ and $\hat{D}-\hat{C} \hat{A}^{-1} \hat{B}=0$. Hence, by using the matrix inversion lemma as follows. If $X_{1}, X_{3}$, and $X_{3}^{-1}+X_{4} X_{1}^{-1} X_{2}$ are nonsingular matrices, then

$$
\begin{aligned}
X_{1}^{-1}-X_{1}^{-1} X_{2}\left(X_{3}^{-1}+X_{4} X_{1}^{-1} X_{2}\right)^{-1} X_{4} X_{1}^{-1} \\
=\left(X_{1}+X_{2} X_{3} X_{4}\right)^{-1}
\end{aligned}
$$

we have

$$
\begin{aligned}
\operatorname{det} A_{c 22}= & \operatorname{det}\left[\hat{A}+\hat{B}(I-D \hat{D})^{-1} D \hat{C}\right] \\
= & \operatorname{det}\left[\hat{A}\left\{I+\hat{A}^{-1} \hat{B}(I-D \hat{C} \hat{A}-1 \hat{B})^{-1} D \hat{C}\right\}\right] \\
= & \operatorname{det}\left[\hat{A}\left(I-\hat{A}^{-1} \hat{B} D \hat{C}\right)^{-1}\right] \\
\neq & 0 \\
A_{c 12} A_{c 22}^{-1} A_{c 21}= & B(I-\hat{D} D)^{-1} \hat{C} \\
& \times\left(I-\hat{A}^{-1} \hat{B} D \hat{C}\right) \hat{A}^{-1} \hat{B}(I-D \hat{D})^{-1} C \\
= & B(I-\hat{D} D)^{-1}(I-\hat{D} D) \hat{D}(I-D \hat{D})^{-1} C \\
= & B(I-\hat{D} D)^{-1} \hat{D} C .
\end{aligned}
$$

Therefore

$$
\begin{aligned}
\operatorname{det} A_{c} & =\operatorname{det} A_{c 22} \operatorname{det}\left[A_{c 11}-A_{c 12} A_{c 22}^{-1} A_{c 21}\right] \\
& =\operatorname{det} A_{c 22} \operatorname{det} A
\end{aligned}
$$

Since the closed-loop system is asymptotically stable, $A_{c}$ does not have any zero eigenvalues. Hence, $\operatorname{det} A_{c} \neq 0$. Therefore, from (19), $\operatorname{det} A \neq 0$.

(Sufficiency) Under the condition that $\operatorname{det} A \neq 0$, we will show that the controller given by (13) is a washout controller which makes (15) asymptotically stable. Let us suppose that $F$ and $L$ be chosen from $\mathcal{S}$. Then, $I+Q D$ is nonsingular, and the feedback controller is given by

$$
\begin{aligned}
{\left[\begin{array}{ll}
\hat{A} & \hat{B} \\
\hat{C} & \hat{D}
\end{array}\right]=} & \mathcal{F}_{l}(J, Q) \\
= & {\left[\begin{array}{cc}
A+B F+L C+L D F & -L \\
F & 0
\end{array}\right] } \\
& +\left[\begin{array}{cc}
B+L D \\
I
\end{array}\right] Q(I+D Q)^{-1}\left[\begin{array}{ll}
-C-D F & I
\end{array}\right] \\
= & {\left[\begin{array}{cc}
R_{1}-R_{2}(I+D Q)^{-1} D R_{3} & R_{2}(I+D Q)^{-1} \\
(I+Q D)^{-1} R_{3} & Q(I+D Q)^{-1}
\end{array}\right] }
\end{aligned}
$$

where

$$
\begin{aligned}
& R_{1}=A+B F+L C-B Q C \\
& R_{2}=B Q-L \\
& R_{3}=F-Q C .
\end{aligned}
$$

By using $\hat{A}, \hat{B}, \hat{C}, \hat{D}$ in (20) and the matrix inversion lemma (17), we have

$$
\begin{aligned}
(I-\hat{D} D)^{-1}= & \left(I-Q(I+D Q)^{-1} D\right)^{-1} \\
= & \left((I+Q D)^{-1}\right)^{-1} \\
= & I+Q D \\
(I-D \hat{D})^{-1}= & \left(I-D Q(I+D Q)^{-1}\right)^{-1} \\
= & \left((I+D Q)^{-1}\right)^{-1} \\
= & I+D Q \\
A_{c 11}= & A+B(I-\hat{D} D)^{-1} \hat{D} C \\
= & A+B(I+Q D) Q(I+D Q)^{-1} C \\
= & A+B Q C, \\
A_{c 12}= & B(I-\hat{D} D)^{-1} \hat{C} \\
= & B(I+Q D)(I+Q D)^{-1} R_{3} \\
= & B R_{3} \\
A_{c 21}= & B(I-D \hat{D})^{-1} C \\
= & R_{2}(I+D Q)^{-1}(I+D Q) C \\
= & R_{2} C \\
& \hat{A}+\hat{B}(I-D \hat{D})^{-1} D \hat{C} \\
= & R_{1}-R_{2}(I+D Q)^{-1} D R_{3} \\
& +R_{2}(I+D Q)^{-1}(I+D Q) \\
= & R_{1} .
\end{aligned}
$$


Moreover, a similarity transformation of $A_{c}$ with the matrix

$$
T=\left[\begin{array}{ll}
I & 0 \\
I & I
\end{array}\right]
$$

is given by

$$
\begin{aligned}
T^{-1} A_{c} T & =\left[\begin{array}{cc}
I & 0 \\
-I & I
\end{array}\right]\left[\begin{array}{cc}
A+B Q C & B R_{3} \\
R_{2} C & R_{1}
\end{array}\right]\left[\begin{array}{cc}
I & 0 \\
I & I
\end{array}\right] \\
& =\left[\begin{array}{cc}
A+B Q C & B F-B Q C \\
-A-L C & A+L C
\end{array}\right]\left[\begin{array}{ll}
I & 0 \\
I & I
\end{array}\right] \\
& =\left[\begin{array}{cc}
A+B F & B F-B Q C \\
0 & A+L C
\end{array}\right] .
\end{aligned}
$$

Since $A+B F$ and $A+L C$ are stable, so is $A_{c}$. Now, we define $R_{4}=A-L D F$, then it is invertible because

$$
\begin{aligned}
\operatorname{det} R_{4} & =\operatorname{det} A \operatorname{det}\left(I-A^{-1} L D F\right) \\
& =\operatorname{det} A \operatorname{det}(I+Q D) \\
& \neq 0 .
\end{aligned}
$$

Hence

$$
\begin{aligned}
& {\left[\begin{array}{cc}
\hat{A} & \hat{B} \\
\hat{C} & \hat{D}
\end{array}\right]} \\
& =\left[\begin{array}{cc}
(A+B F) R_{4}^{-1}(A+L C) & -(A+B F) R_{4}^{-1} L \\
F R_{4}^{-1}(A+L C) & -F R_{4}^{-1} L
\end{array}\right] .
\end{aligned}
$$

Then, from (21), we have

$$
\begin{aligned}
\operatorname{det} \hat{A}= & \operatorname{det}\left[(A+B F) R_{4}^{-1}(A+L C)\right] \neq 0 \\
\hat{D}-\hat{C} \hat{A}^{-1} \hat{B}= & -F R_{4}^{-1} L+F R_{4}^{-1}(A+L C) \\
& \times\left\{(A+B F) R_{4}^{-1}(A+L C)\right\}^{-1} \\
& \times(A+B F) R_{4}^{-1} L \\
= & -F R_{4}^{-1} L+F R_{4}^{-1} L \\
= & 0 .
\end{aligned}
$$

Therefore, we conclude that (13) gives a continuous-time washout controller.

Since poles of the closed-loop system are eigenvalues of $A+$ $B F$ and $A+L C$ in the case of the washout controller, the pole of the closed-loop system can be arbitrarily placed by selecting matrices $F$ and $L$.

\section{B. Discrete-Time Washout Controller}

In this subsection, let us suppose that a state-space realization of a discrete-time linear system be given as

$$
\begin{aligned}
x(k+1) & =A x(k)+B u(k) \\
y(k) & =C x(k)+D u(k) .
\end{aligned}
$$

Then, we have the following theorem for the full-order discrete-time washout controller.

Theorem 2: There exists a discrete-time washout controller stabilizing (22) if and only if $I-A$ is nonsingular. Moreover, when the previous condition holds, a set of stabilizing discretetime washout controller is given by

$$
\left[\begin{array}{ll}
\hat{A} & \hat{B} \\
\hat{C} & \hat{D}
\end{array}\right]=\mathcal{F}_{l}\left(J, Q^{\prime}\right)
$$

where $Q^{\prime}=F(I-A)^{-1} L, J$ is given by (14), and $(F, L) \in$ $\mathcal{S}^{\prime}:=\{(F, L) \mid A+B F$ and $A+L C$ are asymptotically stable, and $I+Q^{\prime} D$ is nonsingular $\}$.

Proof: The proof of this theorem is very similar to that of Theorem 1, and hence it is omitted.

Remark 1: When $\operatorname{det}(I-A) \neq 0$ and $\operatorname{det}\left(I+Q^{\prime} D\right) \neq$ $0, R:=I-A+\mathrm{LDF}$ is invertible, because

$$
\begin{aligned}
\operatorname{det} R & =\operatorname{det}(I-A) \operatorname{det}\left[I+(I-A)^{-1} \mathrm{LDF}\right] \\
& =\operatorname{det}(I-A) \operatorname{det}\left(I+Q^{\prime} D\right) \\
& \neq 0 .
\end{aligned}
$$

Then, we have

$$
\begin{aligned}
& \hat{A}=I-(I-A-B F) R^{-1}(I-A-L C) \\
& \hat{B}=-(I-A-B F) R^{-1} L \\
& \hat{C}=F R^{-1}(I-A-L C) \\
& \hat{D}=F R^{-1} L .
\end{aligned}
$$

\section{TUNING OF WASHOUT CONTROLLERS FOR MANUAL OPERATIONS}

In this section, we will propose an iterative design method of washout controllers for operator-support control. Since washout controllers are implemented on digital computers, we design a discrete-time washout controller by using a discrete-time linearized model of manual control systems.

To design a washout controller assisting in manual operations, we need a discrete-time linearized model of the manual control system around the operating point. In general, however, modeling of the manual control system is difficult. Hence, using input/ output data observed from the manual control system, a system identification seems promising to obtain a linear model of the manual control system in the vicinity of its operating point.

First, we will obtain a linear model as an initial model via open-loop identification. Note that a relatively small number of data in the vicinity of the operating point are available for system identification since, without any operator support controller, the measured output of the manual control system oscillates significantly. Therefore, the initial model may contain large modeling errors, and such modeling errors can lead to poor performance of the initial controller.

To reduce the effect of modeling errors, closed-loop identification is implemented to refine the model of the manual control system for the control design. Since the vibration is suppressed by the washout control in closed-loop setting, there are enough data to identify the model of the manual control system. In this paper, we will adopt a closed-loop subspace identification method, i.e., the SSARX method proposed by Jansson [12].

\section{A. SSARX Method [12]}

The SSARX method can be categorized as a direct approach to closed loop identification. In this method, it is assumed that a linear system can be described in the innovations form by the following state space realization:

$$
\begin{aligned}
x(k+1) & =A x(k)+B u(k)+K e(k) \\
y(k) & =C x(k)+D u(k)+e(k)
\end{aligned}
$$


where $e(k) \in \Re^{m}$ is the innovation. Define $\tilde{A}=A-K C$ and $\tilde{B}=B-K D$, and assume that $\tilde{A}$ is stable. From (24), we have

$$
\begin{aligned}
y_{s}(k) & =\tilde{\mathcal{O}} x(k)+\tilde{\mathcal{D}} u_{s}(k)+\tilde{\mathcal{K}} y_{s}(k)+e_{s}(k) \\
& =\tilde{\mathcal{O}} \tilde{\mathcal{L}} p(k)+\tilde{\mathcal{D}} u_{s}(k)+\tilde{\mathcal{K}} y_{s}(k)+v_{s}(k)
\end{aligned}
$$

where

$$
\begin{aligned}
& y_{s}(k)=\left[y(k)^{\mathrm{T}} \cdots y(k+s-1)^{\mathrm{T}}\right]^{\mathrm{T}} \\
& u_{s}(k)=\left[u(k)^{\mathrm{T}} \cdots u(k+s-1)^{\mathrm{T}}\right]^{\mathrm{T}} \\
& e_{s}(k)=\left[e(k)^{\mathrm{T}} \cdots e(k+s-1)^{\mathrm{T}}\right]^{\mathrm{T}} \\
& p(k)=\left[y(k-1)^{\mathrm{T}} \cdots y(k-s)^{\mathrm{T}} u(k-1)^{\mathrm{T}} \cdots u(k-s)^{\mathrm{T}}\right]^{\mathrm{T}} \\
& v_{s}(k)=\tilde{\mathcal{O}} \tilde{A}^{s} x(k-s)+e_{s}(k) \\
& \tilde{\mathcal{O}}=\left[\begin{array}{c}
C \\
C \tilde{A} \\
\vdots \\
C \tilde{A}^{s-1}
\end{array}\right] \\
& \tilde{\mathcal{D}}=\left[\begin{array}{cccc}
D & & & \\
C \tilde{B} & D & & \\
\vdots & & \ddots & \\
C \tilde{A}^{s-2} \tilde{B} & \cdots & C \tilde{B} & D
\end{array}\right]
\end{aligned}
$$

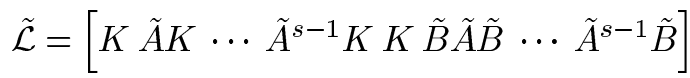

$$
\begin{aligned}
& \tilde{\mathcal{K}}=\left[\begin{array}{cccc}
0 & & & \\
C K & 0 & & \\
\vdots & & \ddots & \\
C \tilde{A}^{s-2} K & \cdots & C K & 0
\end{array}\right] \text {. }
\end{aligned}
$$

Then, the state $x(t)$ is approximated by $\tilde{x}(k):=\tilde{\mathcal{L}} p(k)$. Moreover, when $s$ is sufficiently large, (25) is constructed by stacking ARX models. The procedure of the SSARX method is shown as follows.

1) Estimate a high order ARX model from observed data $u, y$.

2) Estimate $\tilde{\mathcal{D}}$ and $\tilde{\mathcal{K}}$ from coefficients of the estimated high order ARX model. Let $\hat{\tilde{\mathcal{D}}}$ and $\hat{\tilde{\mathcal{K}}}$ denote estimates of $\tilde{\mathcal{D}}$ and $\tilde{\mathcal{K}}$, respectively.

3) From (25), we have

$$
\begin{aligned}
z(k): & =y_{s}(k)-\hat{\tilde{\mathcal{D}}} u_{s}(k)-\hat{\tilde{\mathcal{K}}} y_{s}(k) \\
& \approx \tilde{\mathcal{O}} \tilde{\mathcal{L}} p(k)+v_{s}(k) .
\end{aligned}
$$

By performing canonical correlation analysis on $z(k)$ and $p(k)$, we obtain $\hat{\tilde{\mathcal{L}}}$ which is the estimate of $\tilde{\mathcal{L}}$. Then, the estimate of $\tilde{x}(k)$ is given by $\hat{\tilde{x}}(k)=\hat{\tilde{\mathcal{L}}} p(k)$.

4) Estimate $(A, B, C, D, K)$ and the innovation $e(k)$ from the estimated state $\hat{\tilde{x}}(k)$ and (24).

\section{B. Tuning of Washout Controllers}

Closed-loop identification can be iteratively performed to obtain better models of the manual control system and washout controllers. Now, we summarize a tuning procedure of the washout controller for manual operations via closed-loop identification. The tuning procedure is shown as follows.

1) Identify the initial discrete-time linear model of the manual control system $P_{0}(z)=D_{0}+C_{0}\left(z I-A_{0}\right)^{-1} B_{0}$ via system identification. $i:=0$.

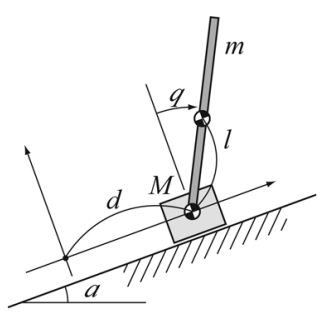

Fig. 3. Inverted pendulum system.

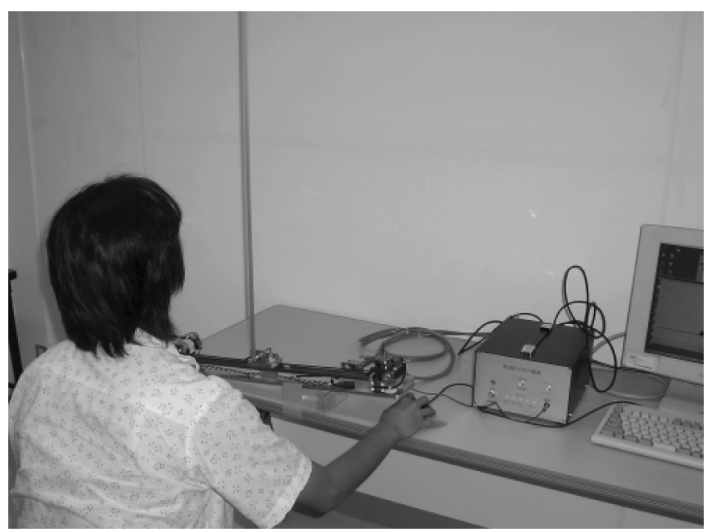

Fig. 4. View of experimental setup.

2) Design the discrete-time washout controller $K_{i}(z)=\hat{D}_{i}+$ $\hat{C}_{i}\left(z I-\hat{A}_{i}\right)^{-1} \hat{B}_{i}$. Then, the washout controller $K_{i}(z)$ are derived from (23) by using the identified model $P_{i}(z)$ and matrices $F$ and $L$ chosen from $\mathcal{S}^{\prime}$.

3) Apply the discrete-time washout controller $K_{i}(z)$ to the manual control system. Go to 4$)$ if necessary.

4) $i:=i+1$. Identify the discrete-time model of the manual control system while applying the discrete-time washout controller $K_{i-1}(z)$. It is given by $P_{i}(z)=D_{i}+C_{i}(z I-$ $\left.A_{i}\right)^{-1} B_{i}$ via the SSARX method. Go to 2 ).

\section{EXPERIMENT With INVERTED PENDULUM SySTEM}

To demonstrate the effectiveness of the proposed technique, we applied it to manual control of the inverted pendulum system on the rail inclined at an unknown angle $\alpha$ from the horizon as illustrated in Fig. 3. The task of a human operator in the manual control system was to stabilize the pendulum by means of moving the cart with the mouse. See Fig. 4. Note that not only the inherent instability of the inverted pendulum in open loop but also the use of the mouse as the input device and the incline of the rail at the unknown $\alpha$ make it difficult for the human operator to stand the inverted pendulum. The length from the joint to the gravity center of the pendulum $l$ is $0.5 \mathrm{~m}$, the mass of the pendulum $m$ is $0.056 \mathrm{~kg}$, the mass of the cart $M$ is $0.235 \mathrm{~kg}$, and the angle of the rail $\alpha$ is $0.18 \mathrm{rad}$. In the experiment, these parameters are assumed to be unknown. In this system, the observed output is the angle of the pendulum $\theta(t)$ and the operating point $\tilde{y}$ is equal to the angle of the rail $\alpha$. Moreover, the sampling time is $20 \mathrm{~ms}$, and the operator-support controller is implemented on a digital computer. 


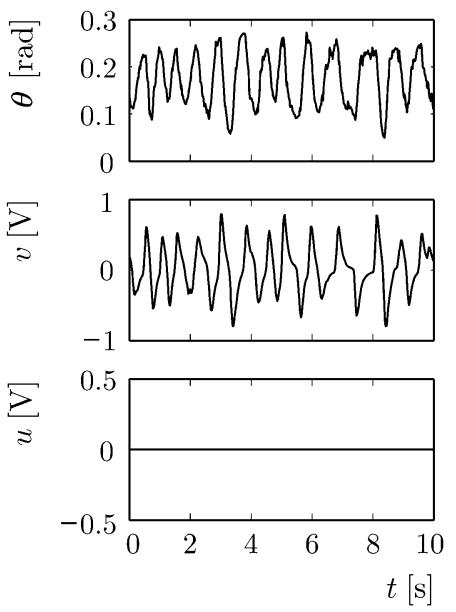

(a)
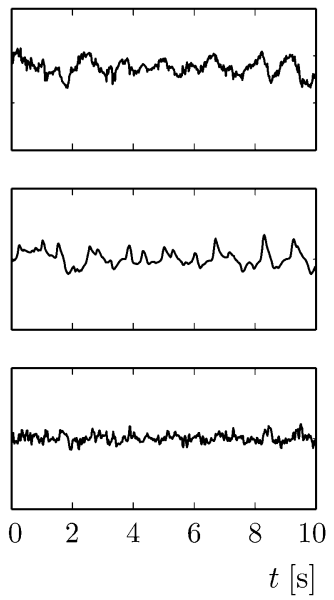

(b)
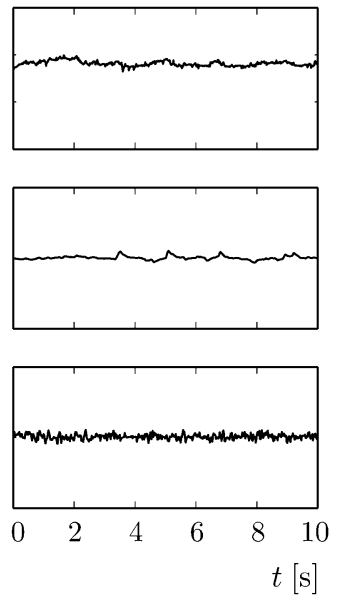

(c)

Fig. 5. Time responses for the inverted pendulum system with (a) only manual control, (b) manual control with the initial washout controller, and (c) manual control with the tuned washout controller.

Fig. 5 shows the time responses of the angle of the pendulum $\theta(t)$, the control input by the human operator $v(t)$, and the control input by the washout controller $u(t)$. Fig. 5(a) is a result obtained with only manual control, whereas Fig. 5(b) has the results for manual control with the initial washout controller, and Fig. 5(c) has the results for manual control with the controller after tuning. In the experiment, the washout controller was redesigned only once. Although the upright state of the pendulum is maintained, as can be seen from Fig. 5(a), vibration occurs. Moreover, there is a great deal of vibration in the control input due to manual manipulation. On the other hand, the washout controller suppresses these vibrations [see Fig. 5(b) and (c)]. Therefore, by using our method, the control input in manual manipulation is suppressed and the load for manual operation is reduced. Moreover, the washout controller after tuning can suppress the vibration in the manual control system to be more effective than the initial controller.

\section{CONCLUSION}

In this paper, we have proposed a new operator-support control scheme for suppressing harmful vibration in manual control systems without disturbing the human operator's manipulation. As support controllers for human operators, we have proposed a washout controller which is a finite-dimensional feedback control method with steady-state blocking zeros, and showed that such a controller can suppress undesirable vibration in a manual control system. Moreover, we have proposed a tuning technique of washout control for manual operations via closed-loop identification. The proposed washout controller is effective for construction of a support system for the human operator.

A future issue is to deal with manual control systems with a time-varying operating point since the proposed scheme assumes that the operating point is constant.

\section{REFERENCES}

[1] C. Riviere, R. S. Rader, and N. V. Thakor, "Adaptive canceling of physiological tremor for improved precision in microsurgery," IEEE Trans. Biomed. Eng., vol. 45, no. 7, pp. 839-846, Jul. 1998.
[2] C. Riviere, W. Ang, and P. Khosla, "Toward active tremor canceling in handheld microsurgical instruments," IEEE Trans. Robot. Automat., vol. 19, no. 5, pp. 793-800, May 2003.

[3] W.-T. Ang, "Active tremor compensation in handheld instrument for microsurgery," Ph.D. dissertation, Robotics Inst., Carnegie Mellon Univ., Pittsburgh, PA, 2004.

[4] K. Pyragas, "Continuous control of chaos by self-controlling feedback," Phys. Lett. A, vol. 170, pp. 421-428, 1992.

[5] A. S. Bazanella, P. V. Kokotovic, and A. S. E. Silva, "On the control of dynamic systems with unknown operating point," Int. J. Control, vol. 73, no. 7, pp. 600-605, 2000.

[6] M. A. Hassouneh, H.-C. Lee, and E. H. Abed, Washout Filters in Feedback Control: Benefits, Limitations and Extensions. College Park, TR: ISR, Univ. of Maryland, 2004, pp. 2004-2016.

[7] M. A. Hassouneh, H.-C. Lee, and E. H. Abed, "Washout filters in feedback control: Benefits, limitations and extensions," in Proc. Amer. Control Conf., 2004, pp. 3950-3955.

[8] T. Takimoto and S. Yamamoto, "Delayed feedback control for vibration suppression in manual control systems," in Proc. SICE Ann. Conf., Aug. 2004, pp. 1498-1501.

[9] H. Kokame, K. Hirata, K. Konishi, and T. Mori, "State difference feedback for stabilizing uncertain steady states of non-linear systems," Int. J. Control, vol. 74, no. 6, pp. 537-546, 2001.

[10] H. Kokame, K. Hirata, K. Konishi, and T. Mori, "Difference feedback can stabilize uncertain steady states," IEEE Trans. Autom. Control, vol. 46, no. 12, pp. 1908-1913, Dec. 2001.

[11] M. S. Saad, M. A. Hassouneh, E. H. Abed, and A. A. Edris, "Delaying instability and voltage collapse in power systems using SVCs with washout filter-aided feedback," in Proc. Amer. Control Conf., 2005, pp. 4357-4362.

[12] M. Jansson, "Subspace identification and ARX modeling," in Proc. 13th IFAC Symp. Syst. Identification, 2003, pp. 1625-1630.

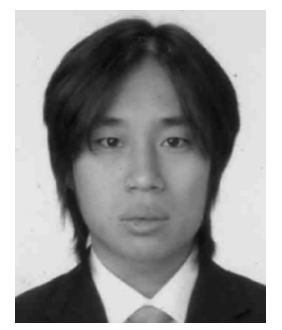

Takashi Takimoto (S'07) was born in Tokyo, Japan, in 1981. He received the B.S. degree from Kitakyushu National College of Technology, Kitakyushnu, Japan, in 2003, and the M.S. degree from Osaka University, Osaka, Japan, in 2005, where he is currently pursuing the Ph.D. degree in engineering.

Mr. Takimoto is a student member of SICE, JSME, and ISCIE. 


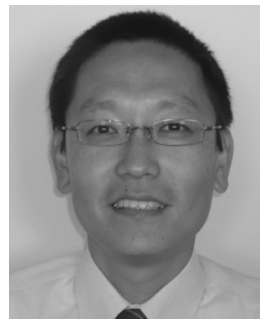

and so on.

Dr. Yamamoto is currently serving as an Associate Editor for the Asian Journal of Control. He is a member of SICE, JSME, and ISCIE.

Shigeru Yamamoto (M'03) was born in Osaka, Japan, in 1964. He received the B.S., M.S., and Ph.D. degrees from Osaka University, Osaka, Japan, in 1987, 1989, and 1996, respectively.

Since 2007, he has been a Professor with Kanazawa University, Ishikawa, Japan. In 1989, he joined Osaka University, as a Research Associate and became an Assistant Professor in 1998, and an Associate Professor in 2000. His research interests include networked control systems, data-oriented controller tuning, human support control systems,

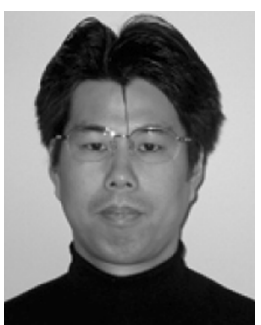

Hiroshi Oku (M'02) received the Ph.D. degree in engineering from the University of Tokyo, Tokyo, Japan, in 2000.

Currently, he is an Associate Professor with the Department of Engineering, Osaka Institute of Technology, Osaka, Japan. In 1998-2000, he was a JSPS Research Fellow. In 2000-2002, He was a Postdoctoral Researcher with the University of Twente. His current research interests include system identification and change detection.

Dr. Oku was a recipient of the Outstanding Paper Award and Sunahara Prize from ISCIE in 2005. He is a member of SICE and ISCIE. 